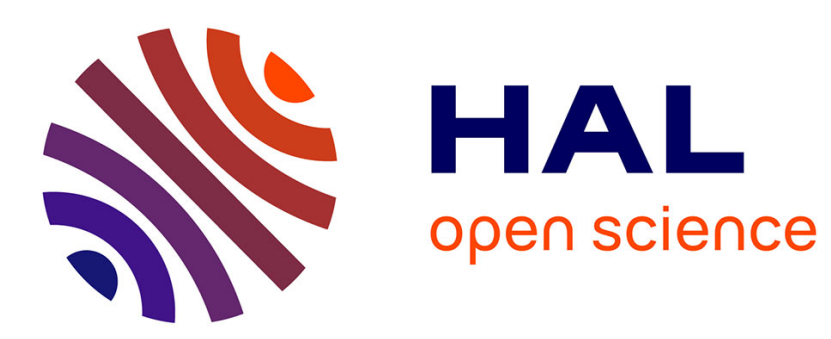

\title{
Verification of Parametric Concurrent Systems with Prioritized FIFO Resource Management
}

\author{
Ahmed Bouajjani, Peter Habermehl, Tomas Vojnar
}

\section{To cite this version:}

Ahmed Bouajjani, Peter Habermehl, Tomas Vojnar. Verification of Parametric Concurrent Systems with Prioritized FIFO Resource Management. 14th International Conference on Concurrency Theory (CONCUR'03), 2003, Marseille, France. pp.172-187. hal-00159537

\section{HAL Id: hal-00159537 https://hal.science/hal-00159537}

Submitted on 3 Jul 2007

HAL is a multi-disciplinary open access archive for the deposit and dissemination of scientific research documents, whether they are published or not. The documents may come from teaching and research institutions in France or abroad, or from public or private research centers.
L'archive ouverte pluridisciplinaire HAL, est destinée au dépôt et à la diffusion de documents scientifiques de niveau recherche, publiés ou non, émanant des établissements d'enseignement et de recherche français ou étrangers, des laboratoires publics ou privés. 


\title{
Verification of Parametric Concurrent Systems with Prioritized FIFO Resource Management ${ }^{\star}$
}

\author{
Ahmed Bouajjani, Peter Habermehl, and Tomáš Vojnar \\ LIAFA, Paris University 7 \\ Case 7014, 2, place Jussieu, 75251 Paris Cedex 05, France \\ e-mail: $\{$ abou, haberm, vojnar\}@liafa.jussieu.fr
}

\begin{abstract}
We consider the problem of parametric verification over a class of systems of processes competing for access to shared resources. We suppose the access to the resources to be controlled according to a FIFO-based policy with a possibility of distinguishing low-priority and high-priority resource requests. We propose a model of the concerned systems based on extended automata with queues. Over this model, we address verification of properties expressed in LTL $\backslash X$ enriched with global process quantification and interpreted on finite as well as fair behaviours of the given systems. In addition, we examine parametric verification of process deadlockability too. By reducing the parametric verification problems to finite-state model checking, we establish several decidability results for different classes of the considered properties and systems (including the special case of systems with the pure FIFO resource management). Moreover, we show that parametric verification against formulae with local process quantification is undecidable in the given context.
\end{abstract}

\section{Introduction}

Managing concurrent access to shared resources is a fundamental problem that appears in many contexts, e.g., operating systems, multithreaded programs, control software, etc. The critical properties to ensure are typically (1) mutual exclusion when exclusive access is required, (2) absence of starvation (a process that requires a resource will eventually get it), and (3) absence of deadlocks. Many different instances of this problem can be defi ned depending on the assumptions on the allowed actions for access to resources and the policies for managing the access to these resources.

In this work, we consider systems with a fi nite number of resources shared by a set of identical processes. These processes can require a set of resources, get access and use the requested resources, and release the used resources. The requests can be of a low-priority or a high-priority level. The access to the resources is managed by a locker according to a FIFO-based policy taking into account the priorities of the requests, i.e. a waiting high-priority request can overtake waiting low-priority ones. As a special case allowing for an optimized treatment, we then examine the situation when no highpriority requests are used, and the locker behaves according to the pure FIFO discipline.

As mentioned later in related work, the above framework is, in particular, inspired by a need to verify the use of shared resources in some of Ericsson's ATM switches. However, the operations for access to shared resources and the resource management policies used are quite natural in general in concurrent applications dealing with shared resources.

\footnotetext{
* This work was supported in part by the European Commission (FET project ADVANCE, contract No. IST-1999-29082).
} 
Verifi cation of the described systems can, of course, be carried out using fi nitestate model-checking if we fi $x$ the number of processes. However, a precise number of processes present in such a system in practice is usually not known in advance, and it is thus crucial to verify that the system behaves correctly for any number of them. This yields a parametric verifi cation problem that is quite nontrivial as we have to deal with an infi nite number of system instances.

The aim of this paper is to study decidability of the described problem for a signifi cant class of properties including the three most important ones given above.

For an abstract description of the concerned systems, we defi ne a model based on extended automata with queues recording the identities of the waiting processes for each resource. Then, we address the verifi cation problem for families of such systems with an arbitrary number of processes (called RTR families - RTR stands for request-takerelease) against formulae of the temporal logic $L T L \backslash X$ with global process quantification. We consider two interpretation domains for the logic: the set of fi nite behaviours (which is natural for safety properties), and the set of fair behaviours (in order to cover liveness properties). In addition, we consider the parametric verifi cation problem of process deadlockability too.

We adopt the approach of fi nding cut-off bounds to show that many interesting parametric verifi cation problems in the given context can be reduced to fi nite-state model checking. This means that given a class of formulae, we prove that deciding whether all systems of a family satisfy a formula is equivalent to deciding whether some fi nite number of systems in the family (each of them having a fi xed number of processes) satisfi es this formula.

When establishing our results, we consider the question whether it is possible to fi nd cut-off bounds that do not depend on the structure of the involved processes and the formula at hand, but only on the number of resources and the number of processes quantifi ed in the formula. Indeed, these numbers are relatively small, especially in comparison to the size of process control automata.

We show that for RTR families where the pure FIFO resource management is used (i.e. no high-priority access to resources is required), parametric verifi cation of fi nite as well as fair behaviour is decidable against all LTL $\backslash X$ formulae with global process quantifi cation. The cut-off bound in the fi nite behaviour case is the number of quantifi ed processes, whereas it is this number plus the number of resources in the fair behaviour case. These bounds lead to practical fi nite-state verifi cation. Furthermore, we show that the verifi cation of process deadlockability is decidable too (where the bound is the number of resources).

On the other hand, for the case of dealing with RTR families that distinguish lowpriority and high-priority requests, we show that-unfortunately-general, structureindependent cut-offs do not exist neither for the interpretation of the considered logic on fi nite nor fair behaviours. However, we show that even for such families, parametric verifi cation of fi nite behaviour is decidable, e.g., against reachability/invariance formulae, and parametric verifi cation of fair behaviour is decidable against formulae with a single quantifi ed process. In this way, we cover, e.g., verifi cation of the (for the given application domain) key properties of mutual exclusion and absence of starvation. For the former case, we even obtain a structure-independent cut-off equal again to the number of quantifi ed processes. For verifi cation of fair behaviour against single process formu- 
lae, no general structure-independent cut-off can be found, but we provide a structuredependent one, and in addition, we determine a signifi cant subclass of RTR families where a structure-independent cut-off for this particular kind of properties does exist. Finally, we show that process deadlockability can be solved in the case of general RTR families via the same (structure-independent) cut-off as in the case of the families not using high-priority requests.

Lastly, we show that although the queues in RTR families are not communication queues, but just waiting queues, and the above decidability results may be established, the model is still quite powerful, and decidability may easily be lost when trying to deal with a bit more complex properties to verify. We illustrate this by proving that parametric fi nite-behaviour verifi cation becomes undecidable (even for families not using high-priority requests) for LTL $\backslash X$ extended with the notion of local process quantification [8] allowing one to examine different processes in different encountered states.

Related Work: There exist several approaches to the parametric verifi cation problem. We can mention, for example, the use of symbolic model checking, (automated) abstraction, or network invariants $[10,1,3,14,11,12]$. The idea of cut-offs has already been used in several contexts $[9,6,7,5]$ too. However, to the best of our knowledge, there is no work covering the class of parametric systems considered here, i.e. parametric resource sharing systems with a prioritized FIFO resource management. The two involved obstacles (parameterization and having multiple queues over an unbounded domain of process identifi ers) seem to complicate the use of any of the known methods. Using cut-offs appears to be the easiest approach here.

The work [5] targets verifi cation of systems with shared resources (and even employs cut-offs), but the system model and the employed proof techniques differ. The involved processes need not be identical, the number of resources is not bounded, but, on the other hand, only two fi xed processes may compete for a given resource, and their requests are served in random order (there are no FIFO queues in [5]). Moreover, some of the properties to be verifi ed we consider here are different from [5] (e.g., we deal with the more realistic notion of weak/strong fairness compared to the unconditional one used there, etc.).

Finally, let us add that our work was originally motivated by [2] that concerns verifi cation of the use of shared resources in Ericsson's AXD 301 ATM switch. In [2] fi nite-state model checking is used to verify some isolated instances of the given parametric system. Then, in the concluding remarks, a need for a more complete, parametric verifi cation is mentioned, which is what our work is aiming at on the level of a general abstract model covering the given application domain.

Outline: We fi rst formalize the notion of RTR families and defi ne the specifi cation logic we use. Then, we present our cut-off results for fi nite and fair behaviour and process deadlockability as well as the undecidability result. Due to space limitations, we provide proof ideas for some of the results only-the complete proofs can be found in [4].

\section{RTR Families}

\subsection{The Model of RTR Families}

Processes in systems of RTR families are controlled by RTR automata. An RTR automaton over a fi nite set of resources is a fi nite automaton with the following kinds 
of actions joint with transitions: skip (denoted by $\tau$-an abstract step not changing resource utilization), request and, when it is the turn, take a set of resources at the low- or high-priority level (rqt/prqt), and, fi nally, release a set of resources ( $r e l$ ).

Let us, however, stress that we allow processes to block inside ( $p$ )rqt transitions ${ }^{1}$ while waiting for the requested resources to be available for them. Therefore, a single (p)rqt transition in a model semantically corresponds to two transitions, which we denote as ( $p$ )req (request a set of resources) and ( $p$ )take (start using the requested resources when enabled to do so by the locking policy).

Definition 1. An RTR automaton is a 4-tuple $\mathcal{A}=\left(R, Q, q_{0}, T\right)$ where $R$ is a set of resources, $Q$ is a set of control locations, $q_{0} \in Q$ is an initial control location, and $T \subseteq Q \times A \times Q$ is a transition relation over the set of actions $A=\{\tau\} \cup\left\{a\left(R^{\prime}\right) \mid a \in\right.$ $\{$ rqt, prqt, rel $\left.\} \wedge R^{\prime} \neq \emptyset \wedge R^{\prime} \subseteq R\right\}$. The sets $R, Q, T$, and $A$ are nonempty, finite, pairwise disjoint, and disjoint with $\mathbb{N}$.

An RTR family $\mathcal{F}(\mathcal{A})$ over an RTR automaton $\mathcal{A}$ is a set of systems $S_{n}$ consisting of $n \geq 1$ identical processes controlled by $\mathcal{A}$ and identifi ed by elements of $P_{n}=\{1, \ldots, n\}$. (In the following, if no confusion is possible, we usually drop the reference to $\mathcal{A}$.) We denote as $R T R \backslash P$ families the special cases of RTR families whose control automata contain no high-priority request actions.

\subsection{Configurations}

For the rest of the section, let us suppose working with an arbitrary fi xed RTR family $\mathcal{F}$ over an automaton $\mathcal{A}=\left(R, Q, q_{0}, T\right)$ and with a system $S_{n} \in \mathcal{F}$.

To make the semantics of RTR families reflect the fact that processes may block in (p)rqt actions, we extend the set $Q$ of "explicit" control locations to $Q_{t}$ containing a unique internal control location $q_{t}$ for each transition $t \in T$ based on a (p)rqt action. Furthermore, let $T_{t}$ be the set obtained from $T$ by preserving all $\tau$ and rel transitions and splitting each transition $t=\left(q_{1},(\mathrm{p}) \operatorname{rqt}\left(R^{\prime}\right), q_{2}\right) \in T$ to two transitions $t_{1}=\left(q_{1},(\mathrm{p}) \mathrm{req}\left(R^{\prime}\right), q_{t}\right)$ and $t_{2}=\left(q_{t},(\mathrm{p}) \operatorname{take}\left(R^{\prime}\right), q_{2}\right)$.

We defi ne the resource queue alphabet of $S_{n}$ as $\Sigma_{n}=\{s(p) \mid s \in\{\mathrm{r}, \mathrm{pr}, \mathrm{g}, \mathrm{u}\} \wedge p \in$ $\left.P_{n}\right\}$. The meaning is that a process has requested a resource in the low- or high-priority way, it has been granted the resource, or it is already using the resource. A configuration $c$ of $S_{n}$ is then a function $c:\left(P_{n} \rightarrow Q_{t}\right) \cup\left(R \rightarrow \Sigma_{n}^{*}\right)$ that assigns the current control locations to processes and the current content of queues of requests to resources. Let $C_{n}$ be the set of all such confi gurations.

\subsection{Resource Granting and Transition Firing}

We now introduce the locker function $\Lambda$ implementing the considered FIFO resource management policy with low- and high-priority requests. This function is to be applied over confi gurations changed by adding/removing some requests to/from some queues in order to grant all the requests that can be granted wrt. the given strategy in the given situation. Note that in the case of RTR $\backslash \mathrm{P}$ families, the resource management policy can be considered the pure FIFO policy.

A high-priority request is granted iff none of the needed resources is in use by or granted to any process, nor it is subject to any sooner raised, but not yet granted,

\footnotetext{
${ }^{1}$ We use (p)rqt when addressing both rqt as well as prqt transitions.
} 
high-priority request. A low-priority request is granted iff the needed resources are not in use nor granted and they are not subject to any sooner raised request nor any later raised high-priority request that can be granted at the given moment. (High-priority requests that currently cannot be granted do not block sooner raised low-priority requests.) Formally, for $c \in C_{n}$, we defi ne $\Lambda(c)$ to be a confi guration of $G_{r}$ equal to $c$ up to the following for each $r \in R$ :

1. If $c(r)=w_{1} \cdot \operatorname{pr}(p) . w_{2}$ for some $p \in P_{n}, w_{1}, w_{2} \in \Sigma_{n}^{*}$ s.t. $c(p)=q_{t}$ for a certain $t=$ $\left(q_{1}, \operatorname{prqt}\left(R^{\prime}\right), q_{2}\right) \in T$ and for all $r^{\prime} \in R^{\prime}, c\left(r^{\prime}\right)=w_{1}^{\prime} \cdot \operatorname{pr}(p) . w_{2}^{\prime}$ with $w_{1}^{\prime} \in\left\{r\left(p^{\prime}\right) \mid\right.$ $\left.p^{\prime} \in P_{n}\right\}^{*}$ and $w_{2}^{\prime} \in \Sigma_{n}^{*}$, we set $\Lambda(c)(r)$ to $\mathrm{g}(p) . w_{1} \cdot w_{2}$.

2. If $c(r)=\mathrm{r}(p) . w$ for some $p \in P_{n}, w \in \Sigma_{n}^{*}$ such that $c(p)=q_{t}$ for a certain $t=$ $\left(q_{1}, \operatorname{rqt}\left(R^{\prime}\right), q_{2}\right) \in T$ and for all $r^{\prime} \in R^{\prime}, c\left(r^{\prime}\right)=r(p) . w^{\prime}$ with $w^{\prime} \in \Sigma_{n}^{*}$, and the premise of case 1 is not satisfi ed for $r$, we set $\Lambda(c)(r)$ to $g(p)$.w.

We defi ne enabling and firing of transitions in processes of $S_{n}$ via a predicate en $\subseteq$ $C_{n} \times T_{t} \times P_{n}$ and a function $t o: C_{n} \times T_{t} \times P_{n} \rightarrow C_{n}$.

For all transitions $t=\left(q_{1}, \tau, q_{2}\right) \in T_{t}$ and $t=\left(q_{1}, a\left(R^{\prime}\right), q_{2}\right) \in T_{t}, a \in\{$ rel, req, preq $\}$, we defi ne $e n(c, t, p) \Leftrightarrow c(p)=q_{1}$. For each transition $t=\left(q_{1},(\mathrm{p}) \operatorname{take}\left(R^{\prime}\right), q_{2}\right) \in T_{t}$, we defi ne $e n(c, t, p) \Leftrightarrow c(p)=q \wedge \forall r \in R^{\prime} \exists w \in \Sigma_{n}^{*}: c(r)=\mathrm{g}(p) . w$. Intuitively, a transition is enabled in some process if the process is at the source control location of the transition and, in the case of $(p)$ take, if the appropriate request has been granted.

Firing of a transition $t=\left(q_{1}, \tau, q_{2}\right) \in T_{t}$ simply changes the control location mapping of $p$ from $q_{1}$ to $q_{2}$, i.e. $t o(c, t, p)=\left(c \backslash\left\{\left(p, q_{1}\right)\right\}\right) \cup\left\{\left(p, q_{2}\right)\right\}$.

Firing of a $(\mathrm{p})$ req transition $t$ corresponds to registering the request in the queues of all the involved resources and going to the internal waiting location of $t$. The locker is applied to (if possible) immediately grant the request. For $t=\left(q_{1},(\mathrm{p}) \operatorname{req}\left(R^{\prime}\right), q_{2}\right) \in T_{t}$, we defi ne $t o(c, t, p)=\Lambda\left(\left(c \backslash c^{-}\right) \cup c^{+}\right)$where $c^{-}=\left\{\left(p, q_{1}\right)\right\} \cup\left\{(r, c(r)) \mid r \in R^{\prime}\right\}$ and $c^{+}=\left\{\left(p, q_{2}\right)\right\} \cup\left\{(r, c(r) .(\mathrm{p}) r(p)) \mid r \in R^{\prime}\right\}$.

For a transition $t=\left(q_{1},(\mathrm{p}) \operatorname{take}\left(R^{\prime}\right), q_{2}\right) \in T_{t}$, we simply change all the appropriate $\mathrm{g}$ queue items to $\mathrm{u}$ items and fi nish the concerned $(\mathrm{p})$ rqt transition, i.e. $t o(c, t, p)=$ $\left(c \backslash c^{-}\right) \cup c^{+}$with $c^{-}$as in the case of (p)req and $c^{+}=\left\{\left(p, q_{2}\right)\right\} \cup\{(r, \mathrm{u}(p) . w) \mid r \in$ $\left.R^{\prime} \wedge c(r)=\mathrm{g}(p) \cdot w\right\}$.

Finally, a rel transition removes the head $u$ items from the queues of the given resources provided they are owned by the given process. The locker is applied to grant all the requests that may become unblocked. Formally, for $t=\left(q_{1}, \operatorname{rel}\left(R^{\prime}\right), q_{2}\right) \in T_{t}$, we fi x $t o(c, t, p)=\Lambda\left(\left(c \backslash c^{-}\right) \cup c^{+}\right)$with $c^{-}=\left\{\left(p, q_{1}\right)\right\} \cup\left\{(r, c(r)) \mid r \in R^{\prime} \wedge \exists w \in \Sigma_{n}^{*}\right.$ : $c(r)=\mathrm{u}(p) . w\}$ and $c^{+}=\left\{\left(p, q_{2}\right)\right\} \cup\left\{(r, w) \mid r \in R^{\prime} \wedge w \in \Sigma_{n}^{*} \wedge c(r)=\mathrm{u}(p) . w\right\}$.

\subsection{Behaviour of Systems of RTR Families}

Let $S_{n}$ be a system of an RTR family $\mathcal{F}$. We defi ne the initial configuration $a_{0}$ of $S_{n}$ to be such that $\forall p \in P_{n}: c_{0}(p)=q_{0}$ and $\forall r \in R: c_{0}(r)=\varepsilon$. By a finite behaviour of $S_{n}$ starting from $c_{1} \in C_{n}$, we understand a sequence $c_{1}\left(p_{1}, t_{1}\right) c_{2} \ldots\left(p_{l}, t_{l}\right) c_{l+1}$ such that for each $i \in\{1, \ldots, l\}$, en $\left(c_{i}, t_{i}, p_{i}\right)$ holds, and $c_{i+1}=t o\left(c_{i}, t_{i}, p_{i}\right)$. If $c_{1}$ is the initial confi guration $c_{0}$, we may drop a reference to it and speak simply about a fi nite behaviour of $S_{n}$. The notion of infinite behaviours of $S_{n}$ can be defi ned in an analogous way. A complete behaviour is then either infi nite or such that it cannot be extended any more. 
We say a complete behaviour is weakly (process) fair iff each process that is eventually always enabled to fi re some transitions, always eventually fi res some transitions. We may call a complete behaviour strongly (process) fair iff each process that is always eventually enabled to fi re some transitions, always eventually fi res some transitions. However, we do not deal with strong fairness in the following as in our model, the notions of strong and weak fairness coincide: Due to the separation of requesting resources and starting to use them and the impossibility of cancelling issued grants of resources, a process cannot temporarily have no enabled transitions without fi ring anything.

For a behaviour $\beta_{n}=c_{1}\left(p_{1}, t_{1}\right) c_{2}\left(p_{2}, t_{2}\right) \ldots$ of a system $S_{n}$ of an RTR family $\mathcal{F}$, we call the confi guration sequence $\pi_{n}=c_{1} c_{2} \ldots$ a path of $S_{n}$ corresponding to $\beta_{n}$ and the transition fi ring sequence $p_{n}=\left(p_{1}, t_{1}\right)\left(p_{2}, t_{2}\right) \ldots$ a run of $S_{n}$ corresponding to $\beta_{n}$. If the behaviour is not important, we do not mention it. We denote $\Pi_{n}^{f i n}, \Pi_{n}^{f i n} \subseteq C_{n}^{+}$, the set of all fi nite paths of $S_{n}$ and $\Pi_{n}^{w f}, \Pi_{n}^{w f} \subseteq C_{n}^{+} \cup C_{n}^{\omega}$, the set of all paths of $S_{n}$ corresponding to complete, weakly fair behaviours.

\section{The Specifi cation Logic}

In this work, we concentrate (with the exception of process deadlockability) on verifi cation of process-oriented, linear-time properties of systems of RTR families. For specifying the properties, we use the below described extension of LTL $\backslash X$, which we denote as MPTL (i.e. temporal logic of many processes). We exclude the next-time operator from our framework because it sometimes allows a certain kind of counting of processes, which is undesirable when trying to limit/reduce the number of processes to be considered in verifi cation.

We extend LTL $\backslash \mathrm{X}$ by global process quantification in a way inspired by ICTL* (see, e.g., [8]) and allowing us to easily reason over systems composed of a parametric number of identical processes. We also allow for an explicit distinction whether a property should hold for all paths or for at least one path out of a given set. Therefore, we introduce a single top-level path quantifier to our formulae. We restrict quantifi cation in the following way: (1) We implicitly require all variables to always refer to distinct processes. (2) We allow only uniformly universal (or uniformly existential) process and path quantifi cation.

Finally, we limit atomic formulae to testing the current control locations of processes. We allow for referring to the internal control locations of request transitions too, which corresponds to asking whether a process has requested some resources, but has not become their user yet.

\subsection{The Syntax of MPTL}

Let $P V, P V \cap \mathbb{N}=\emptyset$, be a set of process variables. We fi rst defi ne the syntax of MPTL path subformulae, which we build from atomic formulae at $(p, q)$ using boolean connectives and the until operator. For $V \subseteq P V$ and $p \in V$, we have:

$$
\varphi(V)::=a t(p, q)|\neg \varphi(V)| \varphi(V) \vee \varphi(V) \mid \varphi(V) \mathcal{u} \varphi(V)
$$

As syntactical sugar, we can then introduce in the usual way formulae like $t t, f f$, $\varphi(V) \wedge \varphi(V), \square \varphi(V)$, or $\diamond \varphi(V)$.

Subsequently, we defi ne the syntax of universal and existential MPTL formulae, which extend MPTL path subformulae by process and path quantifi cation used in a 
uniformly universal or existential way. For $V \subseteq P V$, we have $\Phi_{a}::=\forall V: A \varphi(V)$ and $\Phi_{e}::=\exists V: E \varphi(V)$.

In the rest of the paper, we commonly specify sets of quantifi ed variables by listing their elements in some chosen order. Using MPTL formulae, we can then express, for example, mutual exclusion as $\forall p_{1}, p_{2}: A \square \neg\left(\right.$ at $\left(p_{1}, c s\right) \wedge$ at $\left.\left(p_{2}, c s\right)\right)$ or absence of starvation as $\forall p: A \square($ at $(p, r e q) \Rightarrow \diamond$ at $(p$, use $))$.

\subsection{The Formal Semantics of MPTL}

Suppose working with a set of process variables $P V$. As we require process quantifi ers to always speak about distinct processes, we call a function $v_{n}: P V \rightarrow P_{n}$ a valuation of $P V$ iff it is an injection.

Suppose further that we have a system $S_{n}$ of an RTR family $\mathcal{F}$. Let $\pi_{n} \in C_{n}^{*} \cup C_{n}^{\omega}$ denote a (fi nite or infi nite) path of $S$. For a fi nite (or infi nite) path $\pi_{k}=c_{1} c_{2} \ldots c_{\left|\pi_{n}\right|}$ $\left(\pi_{n}=c_{1} c_{2} \ldots\right)$, let $\pi_{n}^{l}$ denote the suffi x $q c_{l+1} \ldots c_{\left|\pi_{n}\right|}\left(c_{l} c_{l+1} \ldots\right)$ of $\pi_{n}$, respectively. (For a fi nite $\pi_{n}$ with $\left|\pi_{n}\right|<l, \pi_{n}^{l}=\varepsilon$.) Given a path $\pi_{n}$ of $S_{n}$ and a valuation $v_{n}$ of $P V$, we inductively defi ne the semantics of MPTL path subformulae $\varphi(V)$ as follows:

- $\pi_{n}, v_{n}=a t(p, q)$ iff $\pi_{n}=c . \pi_{n}^{\prime}$ and $c\left(\nu_{n}(p)\right)=q$.

- $\pi_{n}, v_{n}=\neg \varphi(V)$ iff $\pi_{n}, v_{n} \not \neq \varphi(V)$.

- $\pi_{n}, v_{n}=\varphi_{1}(V) \vee \varphi_{2}(V)$ iff $\pi_{n}, \nu_{n}=\varphi_{1}(V)$ or $\pi_{n}, \nu_{n}=\varphi_{2}(V)$.

- $\pi_{n}, v_{n} \models \varphi_{1}(V) \mathcal{U} \varphi_{2}(V)$ iff there is $l \geq 1$ such that $\pi_{n}^{l}, v_{n} \models \varphi_{2}(V)$ and for each $k$, $1 \leq k<l, \pi_{n}^{k}, v_{n} \models \varphi_{1}(V)$.

As for any given behaviour $\beta_{n}$ of $S_{n}$, there is a unique path $\pi_{n}$ corresponding to it, we will also sometimes say in the following that $\beta_{n}$ satisfi es or unsatisfi es a formula $\varphi$ meaning that $\pi_{n}$ satisfi es or unsatisfi es $\varphi$. We will call the processes assigned to some process variables by $\mathrm{v}_{n}$ as processes visible in $\pi_{n}$ via $v_{n}$.

Next, let $\Pi_{n} \subseteq C_{n}^{*} \cup C_{n}^{\omega}$ denote any set of paths of $S_{n}$. (Later we concentrate on sets of paths corresponding to all fi nite or fair behaviours.) We defi ne the semantics of MPTL universal and existential formulae as follows:

- $\Pi_{n}=\forall V: A \varphi(V)$ iff for all valuations $v_{n}$ of $P V$ and all $\pi_{n} \in \Pi_{n}, \pi_{n}, \nu_{n}=\varphi(V)$.

- $\Pi_{n} \models \exists V: E \varphi(V)$ iff $\pi_{n}, \nu_{n} \models \varphi(V)$ for some $P V$ valuation $v_{n}$ and some $\pi_{n} \in \Pi_{n}$.

\subsection{Evaluating MPTL over Systems and Families}

Let $S_{n}$ be a system of an RTR family $\mathcal{F}$. Given a universal or existential MPTL formula $\Phi$, we say the fi nite behaviour of $S_{n}$ satisfi es $\Phi$, which we denote by $S_{n} \models_{\text {fin }} \Phi$, iff $\Pi_{n}^{f i n}=\Phi$ holds. We say the weakly fair behaviour of $S_{n}$ satisfi es $\Phi$, which we denote by $S_{n}={ }_{w f} \Phi$, iff $\Pi_{n}^{w f}=\Phi$ holds.

Next, we introduce a notion of MPTL formulae satisfaction over RTR families, in which we allow for specifying the minimum size of the systems to be considered. ${ }^{2} \mathrm{We}$ go on with the chosen uniformity of quantifi cation and for a universal MPTL formula $\Phi_{a}$, an RTR family $\mathcal{F}$, and a lower bound $l$ on the number of processes to be considered,

\footnotetext{
${ }^{2}$ Specifying the minimum size allows one to exclude possibly special behaviours of small systems. Fixing the maximum size would lead to finite-state verification. Although our results could still be used to simplify such verification, we do not discuss this case here.
} 
we defi ne $\mathcal{F}, l \models_{\text {fin }}^{a} \Phi_{a}$ to hold iff $S_{n} \models_{\text {fin }} \Phi_{a}$ holds for all systems $S_{n} \in \mathcal{F}$ with $l \leq n$. Dually, for an existential MPTL formula $\Phi_{e}$, we defi ne $\mathcal{F}, l \models_{\text {fin }} \Phi_{e}$ to hold iff $S_{n} \models_{\text {fin }}$ $\Phi_{e}$ holds for some system $S_{n} \in \mathcal{F}$ with at least $l$ processes. The same notions of MPTL formulae satisfaction over families can be introduced for weakly fair behaviour too.

\section{Verifi cation of Finite Behaviour}

As we have already indicated, one of the problems we examine in this paper is verifi cation of fi nite behaviour of systems of RTR families against correctness requirements expressed in MPTL. In particular, we concentrate on the parametric finite-behaviour verification problem of checking whether $\mathcal{F}, l \models_{\text {fin }}^{a} \Phi_{a}$ holds for a certain RTR family $\mathcal{F}$, a universal MPTL formula $\Phi_{a}$, and a lower bound $l$ on the number of processes to be considered. The problem of checking whether $\mathcal{F}, l={ }_{\text {fin }}^{e} \Phi_{e}$ holds for a certain existential MPTL formula $\Phi_{e}$ is dual, and we will not cover it explicitly in the following.

\subsection{A Cut-Off Result for RTR $\backslash$ P Families}

We fi rst examine the parametric fi nite-behaviour verifi cation problem for the case of RTR $\backslash \mathrm{P}$ families. Let $\Phi_{a} \equiv \forall p_{1}, \ldots, p_{k}: A \varphi\left(p_{1}, \ldots, p_{k}\right)$ be a universal MPTL formula with $k$ globally quantifi ed process variables. We show that for any $\operatorname{RTR} \backslash \mathrm{P}$ family $\mathcal{F}$, the problem of checking $\mathcal{F}, l \models_{\text {fin }}^{a} \Phi_{a}$ can be reduced to simple fi nite-state examination of the system $S_{k} \in \mathcal{F}$ with $k$ processes. At the same time, the processes to be monitored via $p_{1}, \ldots, p_{k}$ may be fi xed to $1, \ldots, k$. We denote the resulting verifi cation problem as checking whether $S_{k}={ }_{\text {fin }} A \varphi(1, \ldots, k)$ holds. Consequently, we can say that, e.g., to verify mutual exclusion in an RTR $\backslash \mathrm{P}$ family $\mathcal{F}$, it suffi ces to verify it for processes 1 and 2 in the system of $\mathcal{F}$ with only these two processes.

Below, we fi rst give a basic cut-off lemma and then we generalize it to the above.

Lemma 1. For an $R T R \backslash P$ family $\mathcal{F}$ and an MPTL path formula $\varphi\left(p_{1}, \ldots, p_{k}\right)$, the following holds for systems of $\mathcal{F}$ :

$$
\forall n \geq k: S_{n} \models_{\text {fin }} \forall p_{1}, \ldots, p_{k}: A \varphi\left(p_{1}, \ldots, p_{k}\right) \Leftrightarrow S_{k}=_{\text {fin }} A \varphi(1, \ldots, k)
$$

Proof. (Sketch) $(\Rightarrow)$ We convert a counterexample behaviour of $S_{k}$ to one of $S_{n}$ by adding some processes and letting them idle at $q_{0} .(\Leftarrow)$ To reduce a counterexample behaviour of $S_{n}$ to one of $S_{k}$, we remove the invisible processes and the transitions fired by them (these processes only restrict the behaviour of others by blocking some resources) and we permute the processes to make $1, \ldots, k$ visible (all processes are initially equal and their names are not signifi cant).

Lemma 1 and properties of MPTL now easily yield the above promised result.

Theorem 1. Let $\mathcal{F}$ be an $R T R \backslash P$ family and let $\Phi_{a} \equiv \forall p_{1}, \ldots, p_{k}: A \varphi\left(p_{1}, \ldots, p_{k}\right)$ be an MPTL formula. Then, checking whether $\mathcal{F}, l \models_{\text {fin }}^{a} \Phi_{a}$ holds is equal to checking whether $S_{k}={ }_{\text {fin }} A \varphi(1, \ldots, k)$ holds.

\subsection{Inexistence of Structure-Independent Cut-Offs for RTR Families}

Unfortunately, as we prove below, for families with prioritized resource management, the same reduction as above cannot be achieved even when we allow the bound to also 


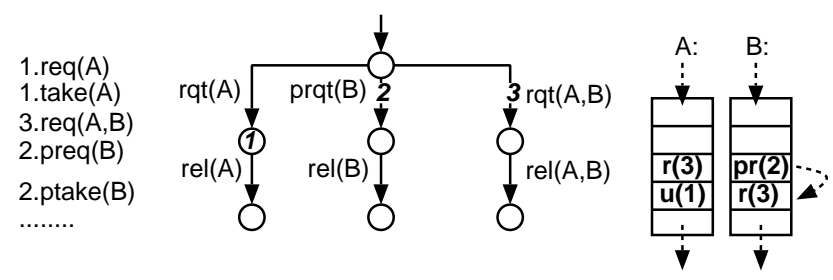

Fig. 1. A scenario problematic for the application of cut-offs (the run from the left is visualized on the RTR automaton and the appropriate resource queues)

depend on the number of available resources and fi $x$ the minimum considered number of processes to one.

Theorem 2. For MPTL formulae $\Phi_{a}$ with $k$ process variables and RTR families $\mathcal{F}$ with $m$ resources, the parametric finite-behaviour verification problem of checking whether $\mathcal{F}, 1 \models_{\text {fin }}^{a} \Phi_{a}$ holds is not, in general, decidable by examining just the systems $S_{1}, \ldots, S_{n} \in$ $\mathcal{F}$ with $n$ being a function of $k$ and/or $m$ only.

Proof. (Idea) In the given framework, we can check whether in some system of the RTR family $\mathcal{F}$ based on the automaton from Fig. 1, some process $p_{1}$ can request A,B before some process $p_{2}$ requests B, but the wish of $p_{2}$ is granted before that of $p_{1}$. As shown in Fig. 1, the above happens in $S_{n} \in \mathcal{F}$ with $n \geq 3$, but not in $S_{2} \in \mathcal{F}$ (the overtaking between visible processes 2 and 3 is impossible without invisible process 1 ). Moreover, when we start extending the $\mathrm{B}$ and $\mathrm{AB}$ branches by more and more pairs of the appropriate (p)rqt/rel actions without extending the A branch, we exclude more than one process to run in these branches via adding $r q t(C) / r e l(C)(\operatorname{rqt}(D) / r e l(D))$ at their beginnings and ends, and we ask whether $p_{1}$ and $p_{2}$ can exhibit more and more overtaking, we will need more and more auxiliary processes in the A branch although $k$ and $m$ will not change.

Despite the above result, there is still some hope that the parametric fi nite-behaviour verifi cation problem for RTR and MPTL can be reduced to fi nite-state model checking. Then, however, the bound on the number of processes would have to also reflect the structure of the RTR automaton of the given family and/or the structure of the formula being examined. We leave the problem in its general form open for future research. Instead, we show below that for certain important subclasses of MPTL, the number of processes to be considered in parametric fi nite-behaviour verifi cation can be fi xed to the number of process variables in the formula at hand as in the RTR $\backslash \mathrm{P}$ case (although the underlying proof construction is more complex). In this way, we cover, among others, mutual exclusion as one of the key properties of the considered class of systems.

\subsection{Cut-Offs for Subclasses of MPTL}

The fir rst subclass of MPTL formulae we consider is the class of invariance and reachability formulae of the form $\Psi_{a}::=\forall V: A \square \psi(V)$ and $\Psi_{e}::=\exists V: E \diamond \psi(V)$ in which $\psi(V)$ is a boolean combination of atomic formulae at $(p, q)$. Mutual exclusion is an example of a property that falls into this class.

Let $\Psi_{a} \equiv \forall p_{1}, \ldots, p_{k}: A \square \psi\left(p_{1}, \ldots, p_{k}\right)$ be an arbitrary invariance MPTL formula with $k$ quantifi ed process variables. We show that for any RTR family $\mathcal{F}$, the parametric 
problem of checking $\mathcal{F}, l==_{\text {fin }}^{a} \Psi_{a}$ can be reduced to the fi nite-state problem of verifying $S_{k} \models_{\text {fin }} A \square \psi(1, \ldots, k)$ with the number of processes fi xed to $k$ and the processes to be monitored via $p_{1}, \ldots, p_{k}$ fi xed to $1, \ldots, k$. As above, we fi rst state a basic cut-off lemma, which we subsequently generalize.

Lemma 2. For any RTR family $\mathcal{F}$ and any nontemporal MPTL path formula $\psi\left(p_{1}, \ldots, p_{k}\right)$, the following holds for systems of $\mathcal{F}$ :

$$
\forall n \geq k: S_{n} \models_{\text {fin }} \forall p_{1}, \ldots, p_{k}: A \square \psi\left(p_{1}, \ldots, p_{k}\right) \Leftrightarrow S_{k} \models_{f i n} A \square \psi(1, \ldots, k)
$$

Proof. (Sketch) We modify the proof of Lemma 1: In the $(\Leftarrow)$ case, to resolve the problem with possibly disallowed overtaking among visible processes that could be enabled only due to some invisible processes blocking some low-priority visible ones (cf. Fig. 1), we postpone fi ring of (p)req transitions to be just before firing of the corresponding ( $p$ )take transitions (or at the very end). Then, since the preserved visible processes release resources as before and do not block them by requests till all originally overtaking requests are served, it can be shown the fi rability of the reduced transition sequence is guaranteed. Moreover, the behaviour is modifi ed in a way invisible for a reachability formula (negation of $\square \psi$ ).

Theorem 3. Let $\mathcal{F}$ be an RTR family and let $\Psi_{a} \equiv \forall p_{1}, \ldots, p_{k}: A \square \psi\left(p_{1}, \ldots, p_{k}\right)$ be an invariance MPTL formula. Then, checking whether $\mathcal{F}, l \models_{\text {fin }}^{a} \Psi_{a}$ holds is equal to checking whether $S_{k} \models_{\text {fin }} A \square \psi(1, \ldots, k)$ holds.

Another subclass of MPTL that can be handled within parametric fi nite-behaviour verifi cation of RTR in the same way as above is the class of formulae in which we allow any of the MPTL operators to be used, but we exclude distinguishing whether a process is at a location from which it can request some resources or whether it has already requested them. Using such formulae, we can, for example, check whether some overtaking among the involved processes is possible or excluded (though not on the level of particular requests). Due to space limitations, we skip a precise formulation of this result here and refer an interested reader to the full version of the paper [4].

\section{Verifi cation of Fair Behaviour}

We next discuss verifi cation of fair behaviour of systems of RTR families against correctness requirements expressed in MPTL. The results presented in this section can be applied for verifi cation of liveness properties, such as absence of starvation, of systems of RTR families. As for fi nite-behaviour verifi cation, we consider the problem of parametric verification of weakly fair behaviour, i.e. checking whether $\mathcal{F}, l \models_{w f}^{a} \Phi_{a}$ holds for an RTR family $\mathcal{F}$, a universal MPTL formula $\Phi_{a}$, and a lower bound $l$ on the number of processes.

We show first that under the pure FIFO resource management, considering up to $m+k$ processes-with $m$ being the number of resources and $k$ the number of visible processes - suffi ces for parametric verifi cation of weakly fair behaviour against any MPTL formulae. By contrast, for prioritized resource management, we prove that (as for fi nite behaviour verifi cation) there does not exist any general, structure-independent cut-off that would allow us to reduce parametric verifi cation of weakly fair behaviour 
to fi nite-state verifi cation. Moreover, we show that, unfortunately, the inexistence of a structure-independent cut-off concerns, among others, also verifi cation of the very important property of absence of starvation. Thus, for the needs of parametric verifi cation of fair behaviour, we subsequently examine in more detail the possibility only sketched in the previous section, i.e. trying to fi nd a cut-off reflecting the structure of the appropriate RTR automaton and/or the structure of the formula.

\subsection{A Cut-Off Result for RTR $\backslash P$ Families}

Let $\mathcal{F}$ be an RTR $\backslash \mathrm{P}$ family with $m$ resources and $\Phi_{a} \equiv \forall p_{1}, \ldots, p_{k}: A \varphi\left(p_{1}, \ldots, p_{k}\right)$ a universal MPTL formula with $k$ process variables. We show that the parametric verifi cation problem of weakly fair behaviour for $\mathcal{F}$ and $\Phi_{a}$ can be reduced to a series of fi nite-state verifi cation tasks in which we do not have to examine any systems of $\mathcal{F}$ with more than $m+k$ processes. The processes to be monitored via $p_{1}, \ldots, p_{k}$ may again be fi xed to $1, \ldots, k$. We denote the thus arising fi nite-state verifi cation tasks as checking whether $S_{n} \models_{w f} A \varphi(1, \ldots, k)$ holds.

As in Section 4, we now fi rst state a basic cut-off lemma and then we generalize it. However, the way we establish the cut-off turns out to be signifi cantly more complex, because lifting a counterexample behaviour from a small system to a big one is now much more involved than previously. To ensure weak process fairness, newly added processes must be allowed to fi re some transitions, but at the same time, this cannot influence the behaviour of the visible processes.

Lemma 3. For systems of an $R T R \backslash P$ family $\mathcal{F}$ with $m$ resources and an MPTL path formula $\varphi\left(p_{1}, \ldots, p_{k}\right)$, the following holds:

$$
\forall n \geq m+k: S_{n} \models_{w f} \forall p_{1}, \ldots, p_{k}: A \varphi\left(p_{1}, \ldots, p_{k}\right) \Leftrightarrow S_{m+k} \models_{w f} A \varphi(1, \ldots, k)
$$

Proof. (Idea) $(\Leftarrow)$ Similar to Lemma 1 . To eventually forever block in $S_{m+k}$ the visible processes that forever block in $S_{n}$, we need at most one invisible process per resource. $\Rightarrow$ ) To extend a counterexample behaviour $\beta_{m+k}$ of $S_{m+k}$ to one of $S_{n}$, we distinguish three cases: (1) If all original processes deadlock in $\beta_{m+k}$, the newly added ones can deadlock too. (2) If all processes run in $\beta_{m+k}$, at least one process may be shown to eventually not use any resource or always eventually release all of them. The new processes can mimic its behaviour. As they regularly do not block any resources, we may interleave them in the non-blocking phases with each other and with the original processes (without influencing the visible ones). (3) The case when some processes get blocked and some run forever in $\beta_{m+k}$ may be split to subcases solvable like (1) or (2).

Now, the theorem generalizing the lemma can be easily obtained by exploiting properties of MPTL. Note, however, that unlike in Theorems 1 and 3, it leads to a necessity of examining several systems, which is due to the difference between the cut-off bound $m+k$ and the number $k$ of visible processes.

Theorem 4. Let $\mathcal{F}$ be an $R T R \backslash P$ family with $m$ resources and let $\Phi_{a} \equiv \forall p_{1}, \ldots, p_{k}$ : $A \varphi\left(p_{1}, \ldots, p_{k}\right)$ be an MPTL formula. Then, checking whether $\mathcal{F}, l \models_{w f}^{a} \Phi_{a}$ holds is equal to checking whether $S_{n} \models_{w f} A \varphi(1, \ldots, k)$ holds for all systems $S_{n} \in \mathcal{F}$ such that $\min (\max (l, k), m+k) \leq n \leq m+k$.

We show in [4] that examining the systems $S_{k}$ (if $l \leq k$ ) and $S_{m+k}$ is necessary for the above result. The question of a potential optimization of the result by not having to examine all the systems between $\max (l, k)$ and $m+k$ remains open for the future, but this does not seem to be a real obstacle to practical applicability of the result. 


\subsection{Absence of Structure-Independent Cut-Offs for RTR families}

In verifi cation of weakly fair behaviour of RTR families against MPTL formulae, we examine complete, usually infi nite behaviours of systems of the considered families. However, to be able to examine such behaviours, we need to examine their fi nite prefi xes as well. Then, Theorem 2 immediately shows that there does not exist any structure independent cut-off allowing us to reduce the given general problem to fi nite-state verifi cation. Moreover, for the case of verifying fair behaviour of RTR families against MPTL formulae, no structure-independent cut-offs exist even for more restricted scenarios than in fi nite behaviour verifi cation. Namely, the query used in the proof of Theorem 2 speaks about two processes. However, below, we give a theorem showing that for the case of parametric verifi cation of weakly fair behaviour, no structure-independent cut-off exists even for single-process MPTL formulae, i.e. formulae having a single process variable and thus speaking about a single visible process. In particular, such a cut-off does not exist for a single-process formula encoding absence of starvation. The theorem is proven in [4] by giving an example family.

Theorem 5. For RTR families $\mathcal{F}$ with $m$ resources and the property of absence of starvation expressed as $\Phi_{a} \equiv \forall p: A \square($ at $(p, r e q) \Rightarrow \diamond$ at $(p$, use $))$, the problem of checking whether $\mathcal{F}, 1 \models_{w f}^{a} \Phi_{a}$ holds is not, in general, decidable by examining just the systems $S_{1}, \ldots, S_{n} \in \mathcal{F}$ with $n$ being a function of $m$ only.

\subsection{A Cut-Off for Single-Process MPTL Formulae}

There is no simple cut-off for verifi cation of weakly fair behaviours of RTR families against single-process MPTL formulae since a lot of invisible processes requesting resources with high priority may be needed to block a visible process. Their number depends on the structure of the control automaton. However, this number can be bounded as shown in this section.

To give the bound we need some defi nitions. Let $\mathcal{F}(\mathcal{A})$ be an RTR family with $m$ resources. The set of control locations $Q_{t}$ of $\mathcal{A}$ is split into two disjoint parts: $Q_{o}$ (all internal control locations and those where processes own at least one resource, without loss of generality a process owns always the same resources at a given control location) and $Q_{n}$ (the others). Let $F=\left|Q_{n}\right|$ ( $F \geq 1$ as $Q_{n}$ contains the initial location $\left.q_{0}\right), C=\left|2^{Q_{o}}\right|=2^{\left|Q_{o}\right|}$ and $M_{C}=C^{C}$. Then, we can defi ne the needed bound as $B_{\mathcal{F}}=$ $C M_{C}\left(M_{C}+1\right)\left(2 F C\left(M_{C}+1\right)\right)^{F}+2 C\left(M_{C}+1\right)+2 m+1$.

The key cut-off lemma below shows that if a formula is true in systems having between $m+1$ and $B_{\mathcal{F}}$ processes, it is also true in systems with more than $B_{\mathcal{F}}$ processes. This and Lemma 5 stating the opposite allows us to reduce the parametric verifi cation problem to verifi cation of systems with up to $B_{\mathcal{F}}$ processes.

Lemma 4. Let $\mathcal{F}$ be an RTR family with $m$ resources and $\varphi(p)$ an MPTL path formula. Then the following holds for systems of $\mathcal{F}$ :

$$
\forall n \geq B_{\mathcal{F}}:\left(\forall n^{\prime}, m+1 \leq n^{\prime} \leq B_{\mathcal{F}}: S_{n^{\prime}}=_{w f} \forall p: A \varphi(p)\right) \Rightarrow S_{n} \models_{w f} \forall p: A \varphi(p)
$$

Proof. (Idea) The full proof is very involved. It is based on the fact that the exact identity of invisible processes is not important, only their number is. The problem of fi nding the bound can then be seen as fi nding a bounded solution of a linear equation system encoding properties of admissible counterexamples, which is possible with a lemma from Linear Integer Programming [13]. 
We now give the counterpart to Lemma 4 whose proof is similar to the proof of the appropriate direction of Lemma 3.

Lemma 5. Let $\mathcal{F}$ be an RTR family and $\varphi(p)$ an MPTL path formula. Then, for systems of $\mathcal{F}$, we have: $\forall n^{\prime} \geq m+1, n \geq n^{\prime}: S_{n} \models_{w f} A \varphi(1) \Rightarrow S_{n^{\prime}} \models_{w f} \forall p: A \varphi(p)$.

We use Lemmas 4 and 5 to give the complete cut-off result for single-process MPTL formulae and weakly fair behaviour of systems of RTR families.

Theorem 6. Let $\mathcal{F}$ be an RTR family with $m$ resources and let $\Phi_{a} \equiv \forall p: A \varphi(p)$ be a single-process MPTL formula. Then, checking $\mathcal{F}, l \models_{w f}^{a} \Phi_{a}$ is equal to checking $S_{n} \models_{w f}$ $A \varphi(1)$ for all $S_{n} \in \mathcal{F}$ with $l \leq n \leq m+1$ or $n=B_{\mathcal{F}}$.

\subsection{Simple RTR Families}

Above, we have shown that parametric verifi cation of weakly fair behaviour of RTR families against single-process MPTL formulae is decidable, but no really simple reduction to fi nite-state verifi cation is possible in general. We now give a restricted subclass of RTR families for which the problem can be solved using a structure-independent cut-off bound.

An RTR family $\mathcal{F}$ is simple if the set of control locations $Q_{n}$ contains only the initial location $q_{0}$ : Processes start from it by requesting some resources (possibly in different ways) and then they may request further resources as well as release some. However, as soon as they release all of their resources, they go back to $q_{0}$. This class is not unrealistic; it corresponds to systems with a single resource-independent computational part surrounded by actions using resources. For this class we show an improved cut-off bound using $2 m+2$ processes, which is better than $B_{\mathcal{F}}$ for $F=1$. This is basically due to the fact that only $m$ invisible processes can be simultaneously in control locations $Q_{o}$.

Theorem 7. Let $\mathcal{F}$ be a simple RTR family with m resources and let $\Phi_{a} \equiv \forall p: A \varphi(p)$ be a single-process MPTL formula. Then, checking $\mathcal{F}, l \models_{w f}^{a} \Phi_{a}$ is equal to checking $S_{n}=_{w f} A \varphi(1)$ for all $S_{n} \in \mathcal{F}$ with $l \leq n \leq m+1$ or $n=2 m+2$.

Notice that an invisible process can freely move among all locations in a subcomponent $Q^{\prime}$ of $\mathcal{A}$ which is strongly connected by $\tau$-transitions. Therefore, Theorem 7 can be generalized to families whose $Q_{n}$ corresponds to such a component. Moreover, the same idea can be used to optimize the general $B_{\mathcal{F}}$ bound.

\section{Process Deadlockability}

Given an RTR family $\mathcal{F}$ and a system $S_{n} \in \mathcal{F}$, we say that a process $p$ is deadlocked in a confi guration $c \in C_{n}$ if there is no confi guration reachable from $c$ from which we could fi re some transition in $p$. As is common for linear-time frameworks, process deadlockability cannot be expressed in MPTL, and so since it is an important property to check for the class of systems we consider, we now provide a specialized (structure-independent) cut-off result for dealing with it.

Theorem 8. Let $\mathcal{F}$ be an RTR family with $m$ resources. For any $l$, the systems $S_{n} \in \mathcal{F}$ with $l \leq n$ are free of process deadlock iff $S_{\max (m, 2)} \in \mathcal{F}$ is.

Proof. (Sketch) We can encounter scenarios where a group of processes is mutually deadlocked due to some circular dependencies in queues of requests, but also situations where a process is deadlocked due to being always inevitably overtaken by processes that keep running and do not even own any resource forever. However, when we (partial- 
ly) replace overtaking by postponed fi ring of requests (cf. Lemma 2), push blocked high-priority requests before the low-priority ones (the former block the latter, but not conversely), and preserve only the running processes that never release all resources simultaneously, we can show that we suffi ce with one (primary) blocked and/or blocking process per resource.

Let us note that the possibility of inevitable overtaking examined in the proof of Theorem 8 as a possible source of process deadlocks in systems of RTR families is stronger than starvation. Starvation arises already when there is a single behaviour in which some process is eventually always being overtaken. Interestingly, as we have shown, inevitable overtaking is much easier to handle than starvation, and we obtain a cut-off bound that cannot be improved even when we restrict ourselves to RTR $\backslash P$ families with no overtaking.

\section{$7 \quad$ RTR Families and Undecidability}

Finally, we discuss an extension of MPTL by local process quantification [8] where processes to be monitored in a behaviour are not fi xed at the beginning, but may be chosen independently in each encountered state. Local process quantifi cation can be added to MPTL by allowing $\forall V^{\prime}: \varphi\left(V \cup V^{\prime}\right)$ to be used in a path formula $\varphi(V)$ with the semantics $\pi_{n}, v_{n}=\forall V^{\prime}: \varphi\left(V \cup V^{\prime}\right)$ iff $\pi_{n}, v_{n}^{\prime}=\varphi\left(V \cup V^{\prime}\right)$ holds for all valuations $v_{n}^{\prime}$ of $P V$ such that $\forall p \in P V \backslash V^{\prime}: v_{n}^{\prime}(p)=v_{n}(p)$. Such a quantifi cation can be used to express, e.g., the global response property $A \square\left(\left(\exists p_{1}:\right.\right.$ at $\left.\left(p_{1}, r e q\right)\right) \Rightarrow \diamond\left(\exists p_{2}:\right.$ at $\left(p_{2}\right.$, resp $\left.\left.)\right)\right)$, which cannot be encoded with global process quantifi ers if the number of processes is not known. Unfortunately, it can be shown that parametric verifi cation of linear-time fi nitebehaviour properties with local process quantifi cation is undecidable even for RTR $\backslash \mathrm{P}$.

Theorem 9. The parametric finite-behaviour verification problem of checking $\mathcal{F}, 1 \models_{\text {fin }}^{a}$ $\Phi_{a}$ for an $R T R \backslash P$ family $\mathcal{F}$ and an MPTL formula $\Phi_{a}$ with local process quantification is undecidable even when the only temporal operators used are $\square$ and $\diamond$ and no temporal operator is in the scope of any local process quantifier.

Proof. (Idea) The proof is done via simulating two-stack push-down automata and is very complex because the queues we work with are not classical communication queues, but only waiting queues.

\section{Conclusions}

In this paper, we have defi ned an abstract model for a signifi cant class of parametric systems of processes competing for access to shared resources under a FIFO resource management with a possibility of distinguishing low- and high-priority requests. The primitives capturing the interaction between processes and resources and the resource management policies considered are natural and inspired by real-life applications. We have established cut-off bounds showing that many practical parametric verifi cation problems (including verifi cation of mutual exclusion, absence of starvation, and process deadlockability) are decidable in this context. The way the obtained results were established is sometimes technically highly involved, which is due to the fact that the considered model is quite powerful and (as we have also shown) positive decidability can easily be lost if verifi cation of a bit more complex properties is considered.

The structure-independent cut-offs we have presented in the paper are small andfor verifi cation of fi nite behaviour and process deadlockability—optimal. They provide 
us with practical decision procedures for the concerned parametric verifi cation problems and, moreover, they can also be used to simplify fi nite-state verifi cation for systems with a given large number of processes.

The structure-dependent cut-off for single-process formulae and verifying the fair behaviour of the general RTR families is quite big and does not yield a really practical decision procedure. One challenging problem is now to optimize this bound. Although we know that no general structure-independent cut-off exists, the bound we have provided is not optimal, and signifi cantly improved cut-offs could be found especially for particular classes of systems as we have already shown for simple RTR families.

Another interesting issue is to improve the decidability bounds. For general RTR families and arbitrary MPTL formulae, decidability of parametric verifi cation of fi nite as well as fair behaviour remains open. So far, we have only shown that these problems cannot be handled via structure-independent cut-offs. Conversely, the question of existence of practically interesting, decidable fragments of MPTL with local process quantifi cation is worth examining too. If no (or no small) cut-off can be found, we could then try to fi nd some adequate abstraction and/or symbolic verifi cation techniques.

Finally, several extensions or variants of the framework can be considered. For example, the questions of nonexclusive access to resources or nonblocking requests can be examined. Moreover, several other locker policies can be considered, e.g., service in random order or a policy where any blocked process can be overtaken. We believe the results presented here and the reasoning used to establish them provide (to a certain degree) a basis for examining such questions.

Acknowledgment: We thank P.A. Abdulla and T. Arts for fruitful discussions.

\section{References}

1. P. Abdulla, A. Bouajjani, B. Jonsson, M. Nilsson. Handling Global Conditions in Parameterized System Verification. In Proc. of CAV'99, LNCS 1633. Springer, 1999.

2. T. Arts, C. Earle, J. Derrick. Verifying Erlang Code: A Resource Locker Case-Study. In Proc. of FME'02, LNCS 2391. Springer, 2002.

3. K. Baukus, S. Bensalem, Y. Lakhnech, K. Stahl. Abstracting WS1S Systems to Verify Parameterized Networks. In Proc. of TACAS 2000, LNCS 1785. Springer, 2000.

4. A. Bouajjani, P. Habermehl, T. Vojnar. Verification of Parametric Concurrent Systems with Prioritized FIFO Resource Management. Full version available at

http://verif.liafa.jussieu.fr/ vojnar/download/concur03.ps.gz

5. E. Emerson, V. Kahlon. Model Checking Large-Scale and Parameterized Resource Allocation Systems. In Proc. of TACAS'02, LNCS 2280. Springer, 2002.

6. E. Emerson, K. Namjoshi. Reasoning about Rings. In Proc. of POPL'95, 1995.

7. E. Emerson, K. Namjoshi. Automatic Verification of Parameterized Synchronous Systems. In Proc. of CAV'96, LNCS 1102. Springer, 1996.

8. E. Emerson, A. Sistla. Utilizing Symmetry when Model Checking under Fairness Assumptions: An Automata-theoretic Approach. ACM Transactions on Programming Languages and Systems, 19(4), 1997.

9. S. German, A. Sistla. Reasoning about Systems with Many Processes. JACM, 39(3), 1992

10. Y. Kesten, O. Maler, M. Marcus, A. Pnueli, E. Shahar. Symbolic Model Checking with Rich Assertional Languages. In Proc. of CAV'97, LNCS 1254, 1997.

11. R. Kurshan, K. McMillan. A Structural Induction Theorem for Processes. Information and Computation, 117(1), 1995.

12. A. Pnueli, S. Ruah, L. Zuck. Automatic Deductive Verification with Invisible Invariants. In Proc. of TACAS 2001, LNCS 2031. Springer, 2001.

13. J. von Zur Gathen, M. Sieveking. A Bound on Solutions of Linear Integer Equalities and Inequalities. Proceedings of the American Mathematical Society, 1978.

14. P. Wolper, V. Lovinfosse. Verifying Properties of Large Sets of Processes with Network Invariants. In Autom. Verifi cation Methods for Finite State Systems, LNCS 407, 1989. Springer. 